\title{
A Novel Method of Adaptive Repetitive Control for Optical Disk Drivers
}

\author{
Kyungbae Chang and Gwitae Park \\ ISRL, Korea University, \\ 1, 5ga Anam-dong Sungbuk-Gu 136-713 Seoul, South Korea \\ \{lslove, gtpark\}@korea.ac.kr \\ http://control.korea.ac.kr
}

\begin{abstract}
In optical disk drives, which support various speeds, it is not avoidable to have a varying periodic disturbance. However, it is still possible to control optical disk drives by using a controller which repeatedly control the drive to change sampling frequency to follow the change of reference period. This paper introduces an adaptive repetitive control method to attenuate the periodic disturbances. The proposed adaptive repetitive control is built with two parts, the repetitive controller and the frequency multiplier. The repetitive controller uses a varying sampler operating at a variable sampling rate maintained at fixed multiple times of the disturbance frequencies and the frequency multiplier generates the varying sampling frequencies based on the disturbance frequency.
\end{abstract}

\section{Introduction}

In this paper, an adaptive repetitive control that can accommodate the varying period disturbance is proposed. Fig. 1 shows the block diagram of the proposed controller, consisting of an actuator, a feedback compensator, an optical sensor, a frequency multiplier, and a repetitive controller. In the diagram, e represents a tracking error, $r$ is a disturbance, and FG stands for the pulse generating signal which has an integer numbers in each period. The pulse generating signal is generated by a spindle motor. The frequency multiplier counts the number of periods of FG. This frequency multiplier produces the fixed number of cycles for each varying period of the FG signal. The repetitive controller uses a generated sampling frequency per period. In the system, there is no need of adjusting sampling time, because the generated sampling period is always synchronized with the period of the disturbance. The number of the sampling point has the same value in case that the period is not considered. sw1 and sw2 are the track search functions controlled by firmware. The details of the frequency multiplier, the repetitive controller, and the search operation are introduced in the following sections.

\subsection{Frequency Multiplier}

Most optical disk driver systems have FG signals, generated by the spindle motor. The FG signal has the integer numbers per each rotation period. The rotation period is relative to the disturbance and the eccentricity period. In this paper, FG signals are used to estimate the period of the disturbance signal. To generate multiple clocks by 


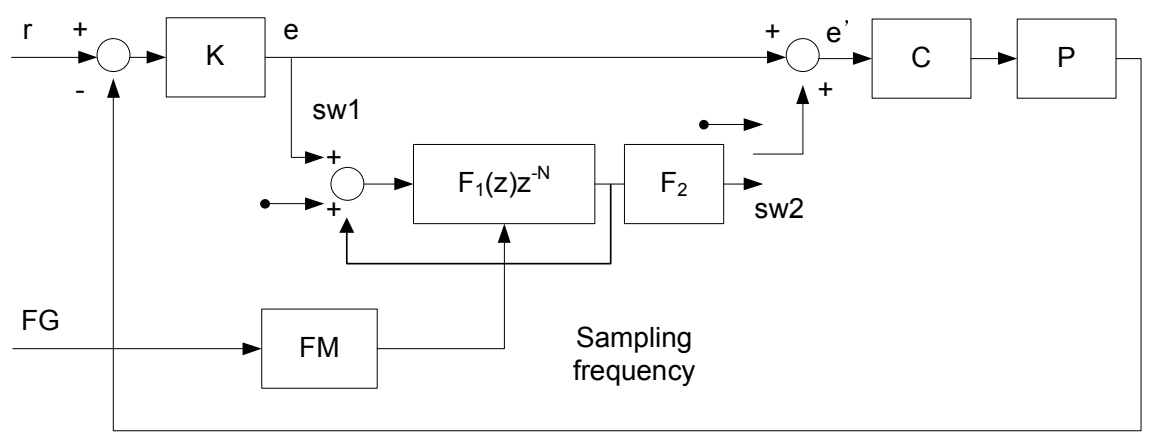

Fig. 1. Adaptive repetitive control system

using an FG signal, the period of the FG signal is estimated followed by dividing the FG signal by integer number. In a digital system, a simple way of measuring the duration of a signal is to count the number of pulses from a source at much higher frequency than the measured signal. The higher frequency is used, the better accuracy of the measurement can be achieved.

Fig. 2 illustrates the timing diagram of the frequency multiplier. The sampling clock of this block is the fixed clock of the existing digital controller. Since the fixed sampling frequency of the existing digital controller is an even higher frequency than the FG signal, it is possible to generate multiple frequencies. This block counts the duration between the rising edges, falling edges, or the edges of the FG signal with the fixed frequency. The counted value is $M$, and $M$ is divided by $L$ which is the defined number of each period. Then the matched counter counts until the match counter reaches the value of M/L, and this block generates the pulse. This pulse is the varying sampling clock with the varying sampling frequency. So the $\mathrm{N}$ samples of the repetitive controller are expressed as;

$$
N=L \times N_{F G}
$$

$\mathrm{N}_{\mathrm{FG}}$ is the number of $\mathrm{FG}$ signals in each rotation period. $\mathrm{L}$ is the defined data, the number per the FG signal. The $\mathrm{N}$ samples always have the same number per each period. In this case, the maximum error period $T_{e}$ of the generated sampling frequency is expressed as;

$$
T_{e}=T_{s} \times(L-1)
$$

If the sampling period $\mathrm{T}_{\mathrm{s}}$ is small enough, the change of the controller's sampling times is small. For example, in case of $\mathrm{T}_{\mathrm{e}}=39.2 \mathrm{uSec}$ for $\mathrm{T}_{\mathrm{s}}=5.6 \mathrm{uSec}, \mathrm{L}=8$, and 6.25 $\mathrm{mSec}$ of the disturbance period, the change deviation is $0.63 \%$. So the effect of the error period is not noticeable.

\subsection{Adaptive Repetitive Controller}

The previous period $\mathrm{M}$ of the FG signal is used instead of the current period $\mathrm{M}+\Delta$. When the current speed is changed into another speed, there is the difference between 


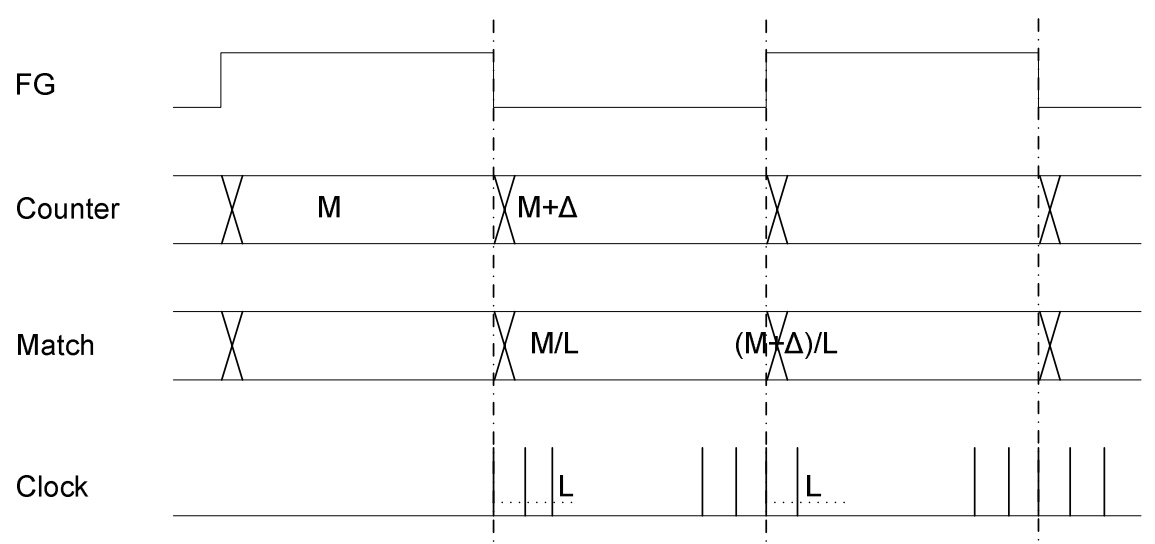

Fig. 2. Timing diagram of the Frequency Multiplier (FM)

the above two periods. Assume that we have a previously recorded waveform e(i) in one revolution with $\mathrm{N}$ equally spaced samples per revolution, $\mathrm{N}=0$ to $\mathrm{N}-1$. Then $\mathrm{e}(\mathrm{i})$ can be decomposed into sinusoidal components. The varying period disturbance can be expressed as the following form:

$$
e(i)=m \sin \left(2 \pi f \frac{i}{N}+\phi\right)
$$

Where, $\mathrm{m}$ and $\phi$ are unknown amplitude and phase. According to the standard of optical disks, $\mathrm{m}$ is usually less than 280um. In CAV mode, when the speed is changed from $24 \mathrm{x}$ to $48 \mathrm{x}$, the average change of the FG period is $1.25 \%$. This indicates that the difference between the previous period and the current one is $1.25 \%$. The frequency multiplier generates the sampling frequency per each pulse. The change deviation $\delta$ between the edges is characterized by

$$
\delta=\frac{T_{F G} \times 0.0125}{T_{e c c}}\left(T_{F G}=T_{e c c} / N_{F G}\right)
$$

$\mathrm{T}_{\text {ecc }}$ is the period of the eccentric disturbance. For example, $\delta=0.14 \%$ for $\mathrm{T}_{\text {ecc }}=1.25 \mathrm{mSec}$ and NFG $=9$. Therefore, in practice, the system stability is possible to be maintained without any change to the repetitive controller's parameters in all cases.

For the repetitive control, it is very important to keep the control period synchronized to the signal period. The frequency multiplier generates the same number of frequencies which has the pulse per each period and is synchronized to the periodic disturbance. Then the repetitive controller is executed at the generated sampling frequency from the frequency multiplier. Hence there is no need to keep the control period synchronized to the periodic disturbance.

In this paper, F1 is chosen as a band pass filter with linear phase characteristic in center frequency. The filter F1 is a 3rd order filter. However, the band pass filter has some of the phase in other frequencies. The sampling frequency of the filter F1 uses the varying sampling frequency from the frequency multiplier. In digital filter, in case of the coefficient having the same value, the phase characteristic is in proportion to the sampling frequency. The characteristic is shown as following Fig. 3. 


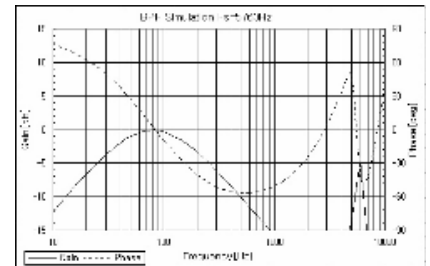

(a) $80 \mathrm{~Hz}(24 \mathrm{x})$

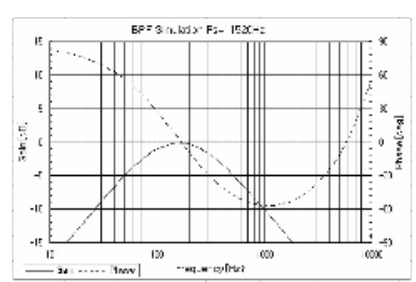

(b) $160 \mathrm{~Hz}(48 \mathrm{x})$

Fig. 3. Gain, Phase characteristics of filter for band limitation

\section{Conclusion}

This paper introduces the control method of varying periodic disturbances for a trackfollowing servo system of an optical disk driver. An adaptive repetitive control is proposed and implemented on the real optical disk driver system. The frequency multiplier is used to generate the adaptive sampling frequency and to exactly synchronize the disturbance and the control period. The result of experiments verifies the effectiveness of the proposed adaptive repetitive controller.

A discrete system is built to track a variable periodic signal with varying sampling time. The proposed adaptive repetitive control is useful enough to be applied to the real world optical disk drive products.

\section{References}

1. Z.Cao and Gerard F. Ledwich. "Adaptive repetitive control to track variable periodic signals with fixed sampling rate", IEEE ASME Transactions on Mechatronics. Vol.7, No.3 Sep, 2002, pp378-384.

2. G.M Dotsch and Henk T. Smakman, "Adaptive Repetitive Control of a Compact Disc Mechanism", the 34th Conference on Decision \& Control, IEEE 1995, pp1720-1725.

3. T.Y.Doh, J.R.Ryoo, M.J.Chung "Repetitive controller design for track-following servo system of Optical Disk Driver", IEEE AMC2002, 2002.

4. Gerard Ledwich, A. Bolton, "Tracking periodic inputs using sampled compensators" Proc. Inst. Elect. Eng. Pt. D, vol. 138 no. 3, 1991.

5. Tadashi Inoue, "Practical repetitive control system design", 29th Conference on Decision and Control, 1990, pp1673-1678.

6. C.Cosner, G.Anwar, M.Tomizuka "Plug In Repetitive Control for Industrial Robotic Manipluators", IEEE, 1990, pp1970-1975.

7. M.Tomizuka,T.Tsao, and K. Chew, "Discrete-Time Doman Analysis and Synthesis of Repetitive Controller", Proc America of Control Conference, 1988, pp860-866. 